\section{MS5-P18 Crystal structures of amidase Cwp6 and related cell wall protein Cwp8 from Clostridium difficile}

Aleksandra Usenik ${ }^{1}$, Miha Renko ${ }^{1,2}$, Marko Mihelič ${ }^{1,2}$, Gregor Pretnar $^{2}$, Dušan Turk ${ }^{1,2}$

1. Jožef Stefan Institute, Ljubljana, Slovenia

2. Centre of Excellence for Integrated Approaches in Chemistry and Biology of Proteins (CIPKeBiP), Ljubljana, Slovenia

email: aleksandra.usenik@ijs.si

The cell wall of Gram-positive bacteria is a surface, exoskeletal organelle composed of peptidoglycan, secondary polymers and a wide variety of proteins providing its unique structural and functional properties. Apart from covalently anchored LPXTG-like cell wall proteins Gram-positive bacteria also harbour several types of cell wall proteins non-covalently attached via cell wall binding domains in some cases forming surface protein layers (S-layers). In Clostridium difficile 630, one of the most important nosocomial pathogens causing antibiotic-associated diarrhea that can lead to potentially lethal pseudomembranous colitis, there are 29 cell wall proteins (CWPs), including the major S-layer precursor, SlpA, sharing three tandem CWB2 repeats. While it was recently shown that CWB2 repeats mediate non-covalent binding to the anionic polymer PSII, we report here the crystal structures of $C$. difficile cell wall proteins Cwp8 and Cwp6 revealing the structure of the cell wall-anchoring module of CWB2 domains. It consist of three tandem, $\sim 100$ residues long, compact CWB2 domains. Additional structural insight into the molecular organization of $C$. difficile cell wall is provided by showing partial structural similarity of the N-terminal part of Cwp8 to the LMW-SLP truncated derivative. Based on biochemical characterization of Cwp6 and its C-terminal domain structural alignment with PDB deposites, we also conclude that Cwp6 is a zinc-dependent N-acetylmuramoyl-L-alanine amidase belonging to an Amidase_3 (PF01520) family.

Keywords: Gram-positive bacteria, Clostridium difficile, cell wall proteins, S-layer, CWB2 domain, amidase

\section{MS5-P19 Conformational study of Bacteroides thetaiotaomicron dipeptidyl peptidase III \\ Marko Tomin ${ }^{1}$, Sanja Tomić1 ${ }^{1}$ Igor Sabljić ${ }^{1}$}

\section{Ruđer Bošković Institute}

email: marko.tomin@irb.hr

Dipeptidyl peptidase III isolated from Bacteroides thetaiotaomicron, Bt-DPP3, is a two-domain zinc exopeptidase from M49 family. Members of this family, characterized by their HEXXGH motive, cleave dipeptidyl residues from the N-terminus of their substrates. The crystallographically determined Bt-DPP3 structure $^{1}$, consisting of two domains separated by a wide cleft, strongly resembles 3D structure of the human ortholog despite their low sequence identity ( $23 \%)$.

Our earlier computational study clearly showed that human DPP3 experiences long-range conformational changes in solution ${ }^{2}$. We showed that, among a number of different forms that it can adopt, the compact form is the most stable and enzymatically active. In this work we used classical and accelerated MD to examine the conformational landscape of Bt-DPP3 as well as influence of ligand binding on the protein structure and dynamics. Special emphasis has been placed on the zinc ion coordination flexibility, since the existing data for human DPP3 suggests the high plasticity of the $\mathrm{Zn}^{2+}$ coordination $^{3}$.

1. I. Sabljić, private communication

2. A. Tomić, M. González, S. Tomić, J Chem Inf Model, 52 (2012) 1583-94.

3. A. Tomić, S. Tomić, Dalton trans., 43 (2014) 15503-14.

Keywords: dipeptidyl peptidase III, computational 